\title{
A comment on "Serological survey of SARS-CoV-2 for experimental, domestic, companion and wild animals excludes intermediate hosts of 35 different species of animals"
}

Ning Zhang ${ }^{1}$, Qin $\mathrm{Xu}^{1}$, Ruiyan Zhang $^{1}$, and Jinghui Zhao ${ }^{1}$

${ }^{1}$ Affiliation not available

October 1, 2020

\begin{abstract}
In May 2020, Deng et al. (2020) published their article providing the evidence that no SARS-CoV-2-specific antibodies were detected in 1,914 samples which excluded the possibility of 35 animal species as intermediate host for SARS-CoV-2 in Transboundary and Emerging Diseases. In order to ensure the stability of SARS-CoV-2-specific antibodies in storage serum samples, we strongly suggest that standard serum banks should be established.
\end{abstract}

\section{Letter to the Editors}

Title: A comment on "Serological survey of SARS-CoV-2 for experimental, domestic, companion and wild animals excludes intermediate hosts of 35 different species of animals"

Running Head : Serum banks for serological survey of SARS-CoV-2

Ning Zhang ${ }^{1}$, Qin $\mathrm{Xu}^{2}$, Ruiyan Zhang ${ }^{1}$, Jinghui Zhao ${ }^{3,4}$,

1. Institute of Biopharmaceutical Research, Liaocheng University, Liaocheng 252059, China

2. General Hospital of Xinjiang Military Region, Wrumuqi 830000, China

3. Scientific Research Center, Guilin Medical University, Guilin 541199, China

4. Changchun Sci-Tech University, Changchun 130600, China

\section{* Correspondence:}

Jinghui Zhao, Scientific Research Center, Guilin Medical University, Guilin 541199, China.

E-mail: zhaojinghui8791@hotmail.com

Keywords: Serum bank; SARS-CoV-2; Serological survey

Dear editor,

In May 2020, Deng et al. (2020) published their article providing the evidence that no SARS-CoV-2-specific antibodies were detected in 1,914 samples which excluded the possibility of 35 animal species as intermediate host for SARS-CoV-2 in Transboundary and Emerging Diseases . In order to ensure the stability of SARS$\mathrm{CoV}$-2-specific antibodies in storage serum samples, we strongly suggest that standard serum banks should be established.

The key of this study was the sera samples, which were collected from 35 different species of animals between November 2019 and March 2020. Notably, the background and storage conditions of the 1,914 serum samples 
were not mentioned in the manuscript. To date, there is no evidence that SARS-CoV-2-specific antibody is stable during different storage temperature and time. The stability of the antibody titers to SARS-CoV-2 may influence the results and conclusion. The storage condition of the samples should be introduced during serological survey of SARS-CoV-2 in the future.

The establishment of serum banks should be considered the following two points. First, serum banks should be included standard preservation conditions and sample background. From sera samples collection to coldchain transportation, it should be in accordance with the standard operating procedures. Additionally, the sera samples should be evaluated and formulated exclusion and inclusion criteria before including in the serum bank. Second, the samples should be collected from people, animals and bats from different regions and times. Besides the SARS-CoV-2, the samples from the serum bank should be used for serological studies of other emerging infectious diseases.

Thank you for your attention in considering this comment and we are looking forward to your response.

\section{CONFLICT OF INTEREST}

The authors declare no conflict of interest relevant to this article.

\section{ACKOWLEDEMENTS}

This work was supported by the Foundation of Liaocheng University (318011907), Doctoral Foundation of Liaocheng University (318051738 and 318051827) and Open Project of Shandong Collaborative Innovation Center for Antibody Drugs (CIC-AD1829 and CIC-AD1834)

\section{REFERENCE}

Deng J., Jin Y., Liu Y., Sun J., Hao L., Bai J., Huang T., Lin D., Jin Y., Tian K. Serological survey of SARS-CoV-2 for experimental, domestic, companion and wild animals excludes intermediate hosts of 35 different species of animals. Transbound Emerg Dis . 2020;67(4):1745-1749. doi: 10.1111/tbed.13577. 